\title{
Ultra-Wideband Microwave Ablation Therapy (UMAT)
}

\author{
Erin Colebeck and Erdem Topsakal \\ Department of Electrical and Computer Engineering, Mississippi State University, \\ MS, 39762, United States
}

\begin{abstract}
We propose an alternative microwave ablation therapy, Ultra-wideband Microwave Ablation Therapy (UMAT), that can potentially be used for the treatment of various cancers including liver, kidney, breast, lung, and bone. The technology relies on extremely small size ultra-wideband antennas that can deliver power to the tissue with more than $90 \%$ power transmission efficiency from beginning to the end of the ablation procedure. The resulting ablation technology is far superior to the existing microwave ablation therapies in terms of power usage, ablation time and zones. In order to validate the system, we provide ex vivo animal experiments.
\end{abstract}

Index Terms - Microwave ablation, ultrawideband antennas, cancer treatment

\section{INTRODUCTION}

Historically, radio frequency (RF) ablation has been the workhorse of all ablation procedures for many years. It has mainly been used for the treatment of heart arrhythmias, and liver tumors. The RF ablation generators operate at very low frequencies (mainly $480 \mathrm{kHz}$ ) and the power levels can be as high as $200 \mathrm{~W}$. The procedure requires a ground pad located in the vicinity of the RF probe to create a close circuit between the probe and the ground. There are several issues associated with this procedure. As the tissue heats up, the tissue conductivity significantly drops and creates an open circuit limiting the ablation zone diameter. Therefore, the ablation zones are small ( $\mathrm{mm}$ range) and ablation times are long (more than 10 minutes). In addition, the ground pads can cause skin burns. Because of the small ablation zones, RF is often inadequate to treat many tumors using single probes [1]-[3]. Recently, Microwave (MW) ablation emerged as a new technology with potential to eliminate the problems associated with RF ablation [4]-[9]. In contrast to RF ablation, MW ablation uses higher frequencies $(915 \mathrm{MHz}$ and $2.4 \mathrm{GHz})$ and works on an electromagnetic energy propagation principle. When the microwave power is turned on, an antenna on the MW probe radiates electromagnetic energy into the tissue creating the ablation zone [10]-[17]. As a result, MW ablation can be used for many organs such as lung and bones with higher impedance values where RF ablation would fail [12]. In addition, MW ablation does not rely on ground pads thereby eliminates the potential unwanted skin burns. Also, it has been shown that MW ablation can create much larger ablation zones compared to RF ablation [18]-[21].

Despite many advantages, there are still major problems associated with the current MW ablation systems. These problems are mainly due to the narrowband nature of the antennas used in these systems. In order to eliminate these problems, we propose to utilize ultra-wideband antennas for more efficient and unique MW ablation therapy.

In this study, we have developed very small size ultrawideband antennas that can be utilized for microwave ablation of liver, lung, kidney, breast, and bone. We provide theory behind the antenna design, present return loss results. Finally, we validate the proposed technology using ex vivo pig liver experiments.

\section{CURRENT MICROWAVE ABLATION SYSTEMS}

As mentioned above, the current microwave ablation systems suffer due to narrowband nature of the antenna used in probes. We will present an illustrative example to show how narrow band antennas can degrade the power transmission efficiency. Consider the dipole antenna in fig. 1 (designed for ablation at $2.4 \mathrm{GHz}$ ). It has been embedded into a medium emulating the electrical properties (dielectric constant $-\varepsilon_{\mathrm{r}}$ and conductivity $\sigma)$ of the liver tissue. It is a known fact that temperature changes affect the electrical properties of the tissue. This effect can be seen in [22]. As seen from the table, $\varepsilon_{\mathrm{r}}$ (from 44.98 to 26.76 ) and $\sigma$ (from 1.79 to 1.26 ) significantly drops as the tissue temperature increases from $15{ }^{\circ} \mathrm{C}$ to $98.9{ }^{\circ} \mathrm{C}$. These changes deteriorate the matching of the antenna impedance to the surrounding tissue impedance resulting significant drop in power transmission efficiency. The red curve in fig. 1 is the reflection curve at the start of the ablation procedure. The arrow on the red curve shows the minimum reflection point at $2.4 \mathrm{GHz}$ with $2.5 \%$ reflection and $97.5 \%$ power transmission into the liver. However, as the temperature increases, the red curve approaches to the blue curve shifting
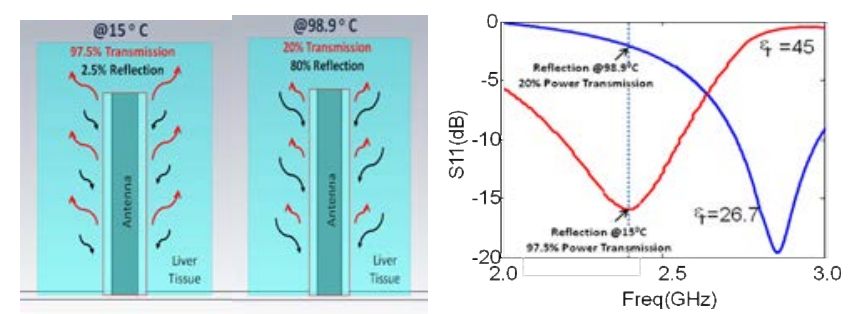

Figure 1. Dipole antenna matched to surrounding liver tissue electrical properties at $15{ }^{\circ} \mathrm{C}$ providing $97.5 \%$ power transmission as opposed to $20 \%$ power transmission when temperature is at $98.9{ }^{\circ} \mathrm{C}$. The dip on the red curve in the plot shows the frequency of operation at $2.4 \mathrm{GHz}$ before the ablation procedure begins.

the minimum reflection point further away from $2.4 \mathrm{GHz}$. At 
the end of the ablation procedure (at $98.9{ }^{\circ} \mathrm{C}$ ), the arrow on the blue curve indicates that only $20 \%$ of the power is transmitted efficiently into the tissue while $80 \%$ is reflected back to the probe. This inefficient power transmission into the tissue results in unwanted ablation regions, higher input power requirements, and longer ablation times.

\section{ULTRA-WIDEBAND MICROWAVE ABLATION PROBE DESIGN}

A slot between two different media behaves like a leaky wave antenna. For a small width of the slot $\mathrm{w}_{\mathrm{s}}$, (compared to the media on either side of the slot) between two different dielectric media the asymptotic value of the complex propagation constant is given by

$$
k_{w}=\beta+\frac{k_{d}^{2}}{2 \beta\left[1-\frac{4}{\pi} \ln \left(\gamma_{e} k_{d} \frac{w_{s}}{8}\right)\right]}
$$

where $\gamma_{\mathrm{e}}$ is the Euler's constant, $k_{d}=\sqrt{k_{2}^{2}-k_{1}^{2}}$, $\beta=\sqrt{\left(k_{2}^{2}+k_{1}^{2}\right) / 2}$, and $k_{i}=k_{o} \sqrt{\varepsilon_{r i}}$ with $i=1,2$ and $k_{o}$ is the free space propagation constant. The rays are launched in the dielectric with lower dielectric constant with an angle $\gamma$ with respect to the slot axis given

$$
\gamma=\cos ^{-1} \sqrt{\frac{\varepsilon_{r 1}+\varepsilon_{r 2}}{2 \varepsilon_{r 2}}} .
$$

The above equation indicates that the direction of the main beam is essentially independent of frequency and we have utilized this phenomenon to design extremely small antennas that can be used for in-body applications. For this, we have exploited the high dielectric constant of human tissues and low permittivity and low loss material like FR4 epoxy on which the antenna is printed. The geometry of the antenna used for ablation is shown in fig.3. It consists of slot which is coiled in a small area $(5.5 \mathrm{~mm} \times 5.5 \mathrm{~mm})$ printed on FR4 substrate and microstrip line printed on the back side which provides the excitation. The antenna is provided with a tapered tip FR4 substrate for ease of insertion into the tissue. The slot, when excited with a suitable microstrip excitation, generates leaky waves over a very large bandwidth. Because the resulting antenna is ultra-wideband in nature, the power transmission efficiency is between $95 \%$ and $99.9 \%$ for all frequencies from $300 \mathrm{MHz}$ to $10 \mathrm{GHz}$ (fig.4).

In this study, our focus will be $915 \mathrm{MHz}, 2.4 \mathrm{GHz}$ and 5.8 GHz. In fig. 3, we show preliminary results from ex-vivo pig liver experiments. These measurements prove that UMAT eliminates the unwanted ablation regions (fig.3, 2nd row 2 nd picture) and allows adjustable ablation zones (fig.3, 2nd row, pictures $3 \& 4$ ) by changing the input frequency. In addition, the power levels and ablation times are significantly lower than previously reported in the literature. The results in figure 3 were generated using $20 \mathrm{~W}$ input power with only 4 minutes of ablation. Also note that in figure 3, we present the first ever MW ablation at $5.8 \mathrm{GHz}$ which is more circular and smaller than the zone at $2.4 \mathrm{GHz}$. We envision that higher frequencies (5.8 GHz or higher) can be used for small tumors that require smaller zones while lower frequencies (2.4 GHz, $915 \mathrm{MHz}$ or lower) can be used for larger tumors.

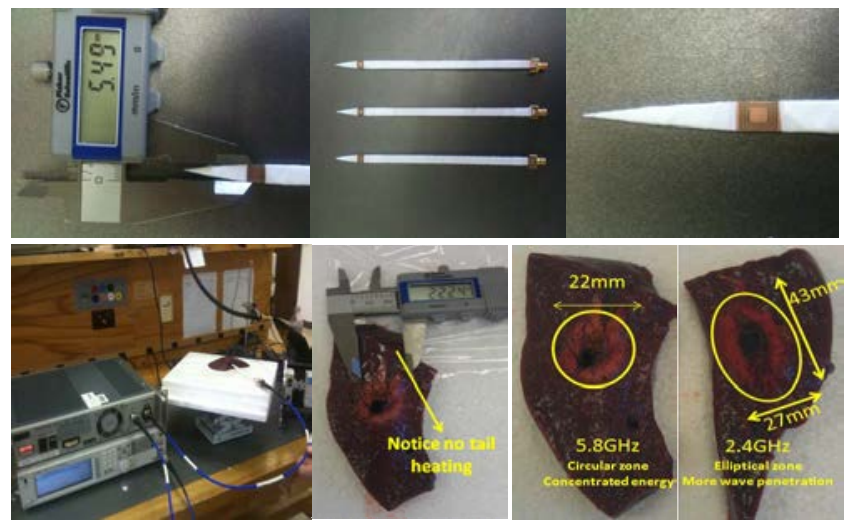

Figure 3. Top pictures show the new ultra-wideband probes. Currently, the antennas are printed on plane substrate for the sake of simplicity, however, in the final prototype, we will modify the antennas to conform on a 14 to 17 gauge probes. The bottom pictures are the measurement setup and the ablation results for 5.8 $\mathrm{GHz}$ and $2.4 \mathrm{GHz}$. Note that the ablation zone at $5.8 \mathrm{GHz}$ is smaller and more circular.

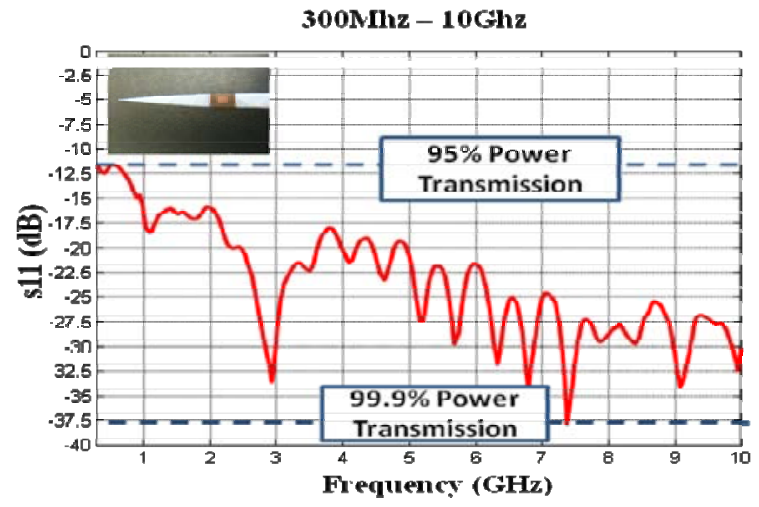

Figure 4. Antenna reflection from $300 \mathrm{MHz}-10 \mathrm{GHz}$.

\section{CONCLUSION}

In order to increase power transmission efficiency, new ultra-wideband probes have been fabricated that function between $300 \mathrm{MHz}$ and $10 \mathrm{GHz}$. Tested at $915 \mathrm{MHz}, 2.4 \mathrm{GHz}$, and $5.8 \mathrm{GHz}$, the ultra-wide band MW ablation probes 
maintain power transmission efficiency between $95 \%$ and $99.9 \%$ while producing optimal ablation zones.

\section{REFERENCES}

[1] [1] Kim YS, Rhim H, Cho OK, Koh Bh, Kim Y, "Ontrahepatic recurrence after percutaneous radiofrequency ablation of heatocellular carcinoma: analysis of the pattern and risk factors." Eur J Radiol 2006; 59: 432-441.

[2] Livraghi T, Goldberg SN, Lazzaroni S, et al., "Hepatocellular carcinoma: radio-frequency ablation of medium and large lesions." Radiology 2000; 214:761768.

[3] Lu DS, Ramen SS, Limanond P, et al., "Influence of large peritumoral vessels on outcome of radiofrequency ablation of liver tumors." J Vasc Interv Radiol 2003; 14:1267-1274.

[4] Lubner MG, Brace CL, Hinshaw JL, Lee FT Jr., "Microwave tumor ablation: mechanism of action, clinical results, and devices." J Vasc Interv Radiol 2010; 21: S192-S203.

[5] Dong B, Liang P, Yu X, et al., "Percutaneous sonographically guided microwave coagulation therapy for hepatocellular carcinoma: results in 234 patients." AJR Am J Roentgenol 2003; 180: 1547-1555.

[6] Castle SM, Salas N, Leveillee RJ., "Initial experience using microwave ablation therapy for renal tumor treatment: 18-month follow-up." Urology 2011; 77: 792797.

[7] Iannitti DA, Martin RC, Simon CJ, et al. "Hepatic tumor ablation with clustered microwave antennae: the US Phase II Trial.” HPB (Oxford) 2007; 9: 120-124.

[8] Kuang M, Lu MD, Xie XY, et al., "Liver cancer: increased microwave delivery to ablation zone with cooled-shaft antenna-experimental and clinical studies". Radiology 2007; 242:914-924.

[9] Liang P, Wang Y., "Microwave ablation of hepatocellular carcinoma". Oncology 2007; 72:124-131.

[10]Brace CL., "Microwave ablation technology: what every user should know." Curr Porbl Diagn Radiol 2009; 38:6167.

[11]Duck F., "Physical properties of tissue: a comprehensive reference book." London: Academic Press; 1980.

[12] Brace CL., "Radiofrequency and microwave ablation of the liver, lung, kidney, and bone: what are the differences?" Curr Probl Diagn Radiol 2009; 38:135-143.

[13] Simon CJ, Dupuy DE, Mayo-Smith WW., "Microwave ablation: principles and applications." Radiographics 2005; 25: S69-S83.

[14] Brace CL, Laaeseke PF, Sampson LA, et al., "Microwave ablation with a single small-gauge triaxial antenna: in vivo porcine model.” Radiology 2007: 435-440.
[15] Hines-Peralta AU, Priani N, Clegg P, et al., "Microwave ablation: results with a $2.45 \mathrm{GHz}$ applicator in ex vivo bovine and in vivo porcine liver." Radiology 2006; 239: 94-102.

[16] Shock SA, Meredith K, Warner TF, et al., "Microwave ablation with loop antenna: in vivo porcine liver model." Radiology 2004; 231:143-149.

[17] Lu MD, Xu HX, Xie XY, et al., "Percutaneous microwave and radiofrequency ablation for hepatocellular carcinoma: a retrospective comparative study." J Gastroenterol 2005; 40:1054-1060.

[18] Yang D, Converse MC, Mahvi DM, Webster JG., "Measurement and analysis of tissue temperature during microwave liver ablation." IEEE Trans Biomed Eng 2007; 54:150-155.

[19] Bhardwaj N, Strickland AD, Ahmad F, Atanesyan L, West K, Lloyd DM., "A comparative histological evaluation of the ablations produced by microwave, cryotherapy, and radiofrequency in the liver." Pathology (Phila) 2009; 41:168-172.

[20] Yu NC, Raman SS, Kim YJ, Lassman C, Chang X, Lu DS., "Microwave liver ablation: influence of hepatic vein size on heat-sink effect in a porcine model." J Vasc Interv Radiol 2008; 19:1087-1092.

[21] Awad MM, Devgan L, Kamel IR, Torbensen M, Choti MA., "Microwave ablation in a hepatic porcine model: correlation of CT and histopahologic findings." HPS (Oxford) 2007; 9:357-362.

[22]Liang P, Wang Y, Yu X, Dong B., "Malignant liver tumors: treatment with percutaneous microwave ablation-complications among cohort of 1136 patients." Radiology 2009; 51:933-940. 